\title{
A CLOSURE THEOREM FOR $\sigma$-COMPACT SUBGROUPS OF LOCALLY COMPACT TOPOLOGICAL GROUPS
}

\author{
TA-SUN WU
}

(Communicated by Jonathan M. Rosenberg)

ABSTRACT. We describe the closure of certain subgroups of a locally compact group.

D. Ž. Djoković proved the following theorem.

Theorem ([1]). Let $G$ be a real Lie group, $A$ a closed subgroup of $G$, and $B$ an analytic subgroup of $G$. We assume that $B$ normalizes $A$ and that $A B$ is closed in $G$. Then we have

$$
B^{-}=(A \cap B)^{-} \cdot B .
$$

In particular, $B$ is closed in $G$ if and only if $A \cap B$ is closed in $G$.

For many interesting applications of the above theorem, we refer to [1] and [2]. In this note, we generalize it into the following theorem.

Theorem. Let $G$ be a locally compact (Hausdorff) topological group. Let $B$ be a $\sigma$-compact subgroup of $G$. Suppose there exists a closed subgroup $A$ of $G$ such that $B$ normalizes $A$ and $B A$ is closed. Then the closure $B^{-}$of $B$ is the group $(A \cap B)^{-} \cdot B$.

Since an analytic subgroup is $\sigma$-compact, Djoković's result is an immediate consequence of the above theorem.

Our proof is simple, using a known categorical argument for topological groups which we state as a lemma (cf. Theorem 5.29 of [4] for a similar result).

Lemma. Let $F$ be a $\sigma$-compact topological group. If there exists a continuous isomorphism $f$ from $F$ onto a locally compact topological group $H$, then $F$ is locally compact and $f$ is a topological isomorphism, i.e. $f$ is an open map.

Proof. First, we show that $F$ is locally compact. Since $F$ is $\sigma$-compact, there exists a sequence of compact subsets $\left\{D_{i}: i=1,2, \ldots\right\}$ of $F$ such that $F=$ $\bigcup_{i=1}^{\infty} D_{i}$. Since $H=f(F)=\bigcup_{i=1}^{\infty} f\left(D_{i}\right), f\left(D_{i}\right)$ has nonvoid interior $f\left(D_{i}\right)^{0}$

Received by the editors July 24, 1989 and, in revised form, October 19, 1989.

1980 Mathematics Subject Classification (1985 Revision). Primary 22A05. 
for some $D_{i}$ by the Baire category theorem. Then $f^{-1}\left(f\left(D_{i}\right)^{0}\right)$ is an open subset of $F$ with compact closure. Therefore $F$ is a locally compact group.

Now, we show that $f$ is an open map. Let $U$ be any compact neighborhood of identity $1_{F}$ of $F$. Let $V$ be a compact neighborhood of $1_{F}$ of $F$ such that $V=V^{-1} \subset V^{2} \subset U$. Since $F$ is $\sigma$-compact, $F=\bigcup_{i=1}^{\infty} x_{i} V$ where $\left\{x_{i}: i=1,2, \ldots\right\}$ is a sequence of elements in $F$. Again, by the Baire category theorem, $f\left(x_{i} V\right)$ has nonvoid interior for some $f\left(x_{i} V\right)$. Since $f(V)=f\left(x_{i}\right)^{-1} \cdot f\left(x_{i} V\right), f(V)$ has a nonvoid interior. Now let $h$ be an interior point of $f(V)$. Let $x=f^{-1}(h)$. Then $f\left(1_{F}\right)=f\left(x^{-1}\right) f(x)=h^{-1} h \in$ $h^{-1} f(V)^{0} \subset f(U)$. Hence $f\left(1_{F}\right)=1_{H} \in f(U)^{0}$, and $f$ is an open map at the identity. Both $F$ and $H$ are homogeneous spaces. It follows that $f$ is an open map. The proof of the lemma is now complete.

Proof of the theorem. Without loss of generality, we assume that $G=B A$. $A$ is a closed normal subgroup of $G$. Since $B$ normalizes $A \cap B, B$ normalizes $(A \cap B)^{-}$. Therefore $B \cdot(A \cap B)^{-}$is a subgroup of $G$. It is straightforward to check that $\left[B \cdot(A \cap B)^{-}\right] \cap A=(A \cap B)^{-}$. Let $\phi$ be the inclusion map from $B \cdot(A \cap B)^{-}$into $G$. Then we have the continuous isomorphism $\phi^{\prime}$ induced by $\phi$ from $B \cdot(A \cap B)^{-} /(A \cap B)^{-}$onto $G / A$. Since $B \cdot(B \cap A)^{-} /(B \cap A)^{-}$is the homomorphic image of $B$, it is $\sigma$-compact. Since $G / A$ is locally compact, $\phi^{\prime}$ is an open map and $B \cdot(A \cap B)^{-} /(A \cap B)^{-}$is locally compact by the above lemma. Since $(A \cap B)^{-}$is locally compact, therefore $B \cdot(A \cap B)^{-}$is locally compact (cf. [3], Theorem 5.25 of [4], or Theorem 2.2 of [5]). Hence $B \cdot(A \cap B)^{-}$is closed. Since $B \subset B \cdot(A \cap B)^{-} \subset B^{-}$, so $B^{-}=B \cdot(A \cap B)^{-}$. The proof is now complete.

\section{REFERENCES}

1. D. Ž. Djoković, A closure theorem for analytic subgroups of real Lie groups, Canad. Math. Bull. 19 (1976), 435-439.

2. $\ldots$ Irreducible connected Lie subgroups of $\mathrm{GL}_{n}(R)$ are closed, Israel J. Math. 28 (1977), 175-176.

3. A. Gleason, Groups without small subgroups, Ann. of Math. 56 (1952), 193-212.

4. E. Hewitt and K. A. Ross, Abstract harmonic analysis, Springer-Verlag, New York, 1963.

5. D. Montgomery and L. Zippin, Topological transformation groups, Interscience, 2nd printing, New York, 1964.

Department of Mathematics, Case Western Reserve University, Cleveland, Ohio 